\title{
DÜBLIN
}

Technological University Dublin ARROW@TU Dublin

2006-01-01

\section{A Compact Wide-band Shorted Folded Antenna}

\author{
Giuseppe Ruvio \\ Technological University Dublin \\ Max Ammann \\ Technological University Dublin, max.ammann@tudublin.ie
}

Follow this and additional works at: https://arrow.tudublin.ie/engschececon

Part of the Electrical and Computer Engineering Commons

\section{Recommended Citation}

Ruvio, G. \& Ammann, M. (2006) A compact wide-band shorted folded antenna. IEEE International Workshop on :Antenna Technology Small Antennas and Novel Metamaterials, pp.84-87. 6-8 March, 2006, doi:10.1109/IWAT.2006.1608981

This Conference Paper is brought to you for free and open access by the School of Electrical and Electronic Engineering at ARROW@TU Dublin. It has been accepted for inclusion in Conference papers by an authorized administrator of ARROW@TU Dublin. For more information, please contact arrow.admin@tudublin.ie, aisling.coyne@tudublin.ie, gerard.connolly@tudublin.ie. Funder: Science Foundatin Ireland 


\title{
A COMPACT WIDE-BAND SHORTED FOLDED ANTENNA
}

\author{
G. Ruvio and M.J. Ammann \\ Centre for Telecommunications Value-Chain Driven Research \\ Dublin Institute of Technology \\ School of Electronic and Communications Engineering \\ Kevin Street - Dublin 8 \\ Ireland \\ Phone: +353-1-4024905/4716 FAX: +353-1-4024690 \\ giuseppe.ruvio@student.dit.ie
}

\section{INTRODUCTION}

Planar monopole antennas are easy to construct from a single metal plate and able to offer attractive performance in terms of impedance bandwidth and radiation properties [1]. However, the necessity of compactness has induced the research community to investigate folded radiation elements [2 and 3]. But, at the same time, by reducing the overall dimensions of planar monopole antennas distortions in the radiation pattern and drawbacks in the impedance bandwidth can be introduced. Special feeding techniques and particular grounding systems have to be designed in order to obtain an acceptable compromise between compact dimensions and interesting performance [4, 5 and 6].

The novel and compact wide-band shorted folded antenna presented in this paper offers a working bandwidth of $4.6 \mathrm{GHz}$ between $1.6 \mathrm{GHz}$ and $7.2 \mathrm{GHz}(\mathrm{IBR}=4.5 ; 1)$. Good radiation properties and acceptable omni-directionality are obtained throughout the operating bandwidth.

The use of a vertical ground plane and a microstrip feeding system permit a dramatic reduction of dimensions when compared to a planar monopole antenna with same characteristics. In this paper, we present another promising bandwidth-enhancement technique, which consists of grounding the radiating element with the vertical ground plane. This solution is shown to only moderately disturb the radiation properties of the antenna.

As a consequence of performance and reduced dimensions, this antenna represents a significant candidate for in-door, mobile and vehicular applications where the coverage of different communication protocols is required.

Numerical simulation have been obtained by CST Microwave Studio and compared to experimental results of the constructed prototype. A good agreement is achieved.

\section{ANTENNA DESIGN}

Figure 1 shows the proposed compact shorted folded antenna together with its geometric parameters and coordination system. The antenna is fed by a $50 \mathrm{ohm}$ microstrip, which is printed on FR4 $1.6 \mathrm{~mm}$ thick substrate $\left(\varepsilon_{\mathrm{r}}=4.4\right)$. The radiating element comprises both a printed part on dielectric and a structure made by folded $0.2 \mathrm{~mm}$ cupper sheet opportunely shaped and attached to the PCB element at the top of the printed part and to the vertical ground plane in the way shown in Fig 1.

The feeding microstrip is offset positioned respect with the centre of the antenna. This enhancement bandwidth technique is very well-known for designing planar monopole antennas and it has been largely demonstrated its effectiveness [6]. Indeed, this offset position of the feeding point allows the excitation of more modes with a consequent and evident improvement in terms of impedance bandwidth.

A double asymmetrical bevel is located at the base of the radiating element. The bevel angle chosen is $\alpha=20^{\circ}$ (Fig. 1). This reshaping of the antenna at its hottest part, just at the insertion of the feeding line is confirmed to be a very powerful tool to shift upward the upper-edge frequency [4]. The distance of the 
ground plane from the bottom edge of the antenna, $f g$, usually plays a significant rule for the impedance bandwidth performance of the antenna as it controls the capacitive coupling of the radiating element with the ground plane. However, when bevels are introduced a mutual dependence between $\alpha$ and $f g$ occurs and a coupled investigation of these two geometric parameters had to be made.

Finally, by grounding the antenna with a horizontal element connecting the vertical the ground plane to the folded radiating element, current distribution is significantly modified and a dramatic enlargement of the impedance bandwidth is obtained [7]. On the other hand, this particular grounding solution perturbs moderately the radiation properties of the antenna.

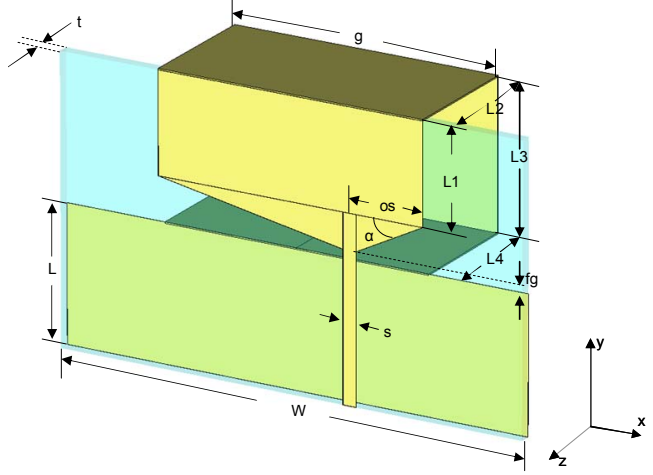

Figure 1 Geometry of the presented antenna. $g=40 \mathrm{~mm}, W=70 \mathrm{~mm}, L=20 \mathrm{~mm}, t=1.6 \mathrm{~mm}, \mathrm{~s}=2 \mathrm{~mm}$, $o s=11 \mathrm{~mm}, g=1.8 \mathrm{~mm}, \alpha=20^{\circ}, L 1=13 \mathrm{~mm}, L 2=$ $21 \mathrm{~mm}, L 3=22 \mathrm{~mm}, L 4=19.4 \mathrm{~mm}$.

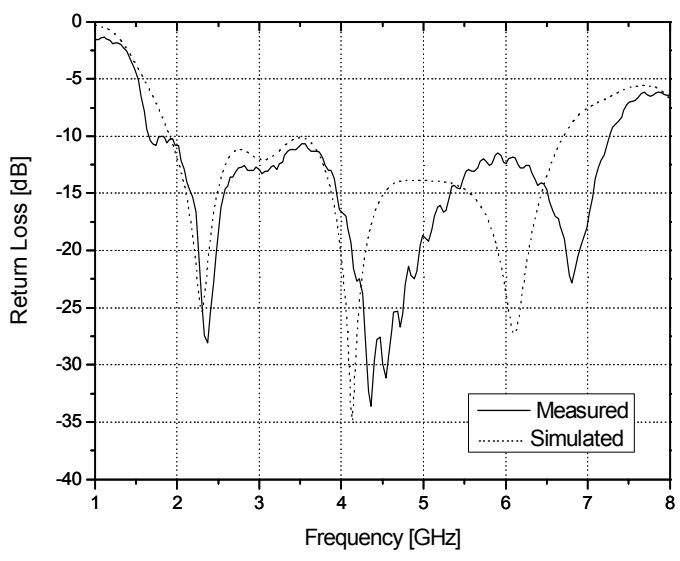

Figure 2 Return loss comparison of measured and

\section{SIMULATED AND MEASURED RESULTS}

The presented antenna offers a $10 \mathrm{~dB}$ return loss bandwidth in the range of frequency $1.6-7.2 \mathrm{GHz}$ $(127 \%)$ as shown in Fig. 2. Simulated results show a slightly narrower bandwidth compared to measurement. However, an acceptable agreement is achieved especially at lower frequencies. This impedance bandwidth allows the coverage of numerous communication protocols including GSM 1800, Personal Communication System (PCS1900), Universal Mobile Communications System (UMTS), the $2.4-\mathrm{GHz} \quad(2400-2484 \mathrm{MHz})$ and $5.2 / 5.8-\mathrm{GHz} \quad(5185-5350 / 5725-5875 \mathrm{MHz})$ bands for WLAN communication, Wireless Local Loop (3.4-3.6 GHz), the US-NII bands and the lower bands allocated to Ultrawideband communications.

This antenna offers appealing radiation properties throughout the working bandwidth. Figures 3,4 and 5 show a comparison of measured and simulated radiation patterns on three different cuts $(x z-, x y$ - and $y z-$ plane respectively) at 3 and $6 \mathrm{GHz}$. Generally a good agreement between numerical and experimental results is achieved. The gain of this antenna is $3.8 \mathrm{dBi}$ and $5.2 \mathrm{dBi}$ respectively at 3 and $6 \mathrm{GHz}$. The radiation patterns displayed in the paper have been normalized to the maximum gain and a $10 \mathrm{~dB} / \mathrm{division}$ scale is adopted in Fig. 3, 4 and 5. Moreover, the cross-polarised E-field component has been included in all the figures. Poor polarisation purity is achieved in the H-plane at lower frequencies. However, the cross-polarised component remains under control on the other cuts. In the two E-planes showed at the two frequencies investigated, it is evident that this antenna works in a hybrid mode due to the combination of a monopolar and a transmission-line (patch) mode. 


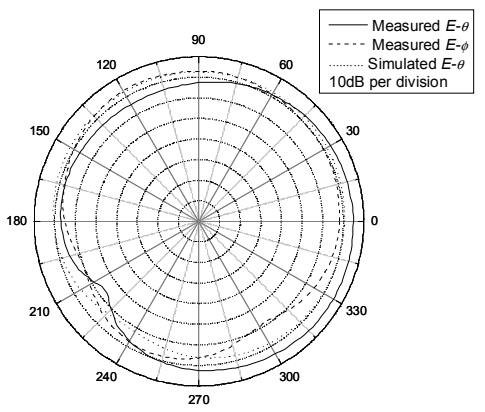

(a)

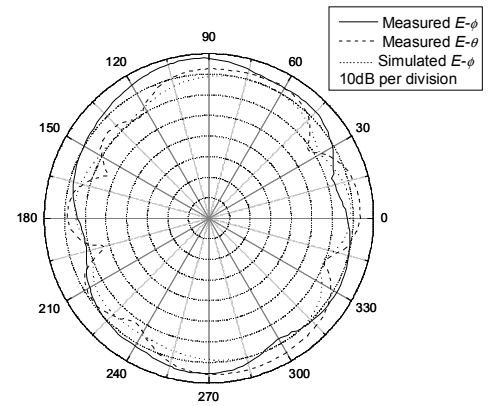

(b)

Figure 3 (a) H-plane (xz-plane) at 3GHz; (b) H-plane (xz-plane) at 6GHz.

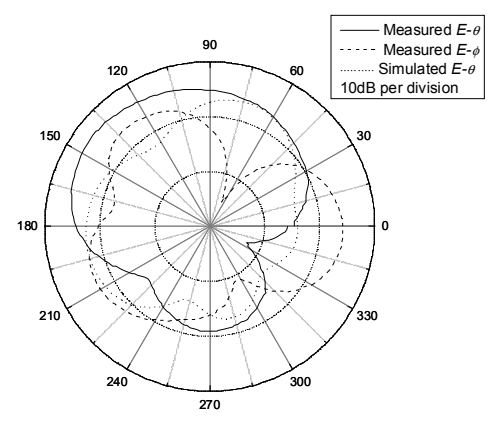

(a)

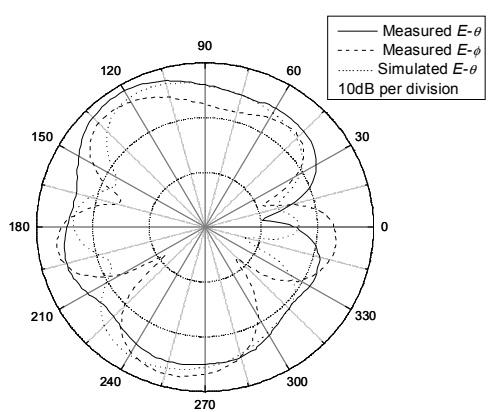

(b)

Figure 4 (a) E-plane $\varphi=0^{\circ}$ (xy-plane) at $3 \mathrm{GHz}$; (b) E-plane $\varphi=0^{\circ}$ (xy-plane) at $6 \mathrm{GHz}$.

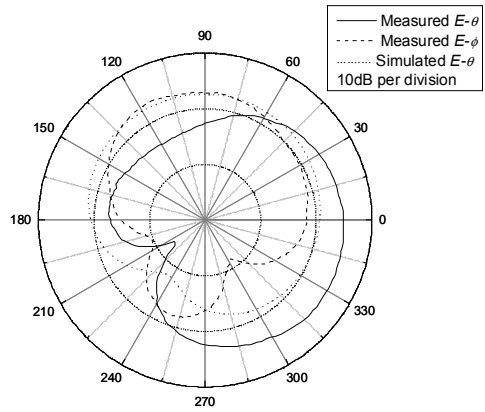

(a)

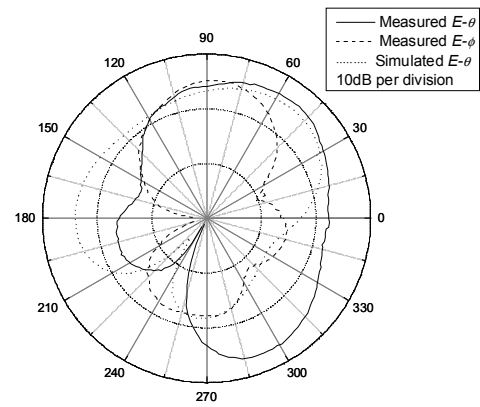

(b)

Figure 5 (a) E-plane $\varphi=90^{\circ}$ (yz-plane) at $3 \mathrm{GHz}$; (b) E-plane $\varphi=90^{\circ}$ (yz-plane) at $6 \mathrm{GHz}$.

\section{CONCLUSIONS}

In this paper a novel folded and grounded antenna has been presented. Simulated and experimental results obtained on a manufactured prototype document its performance. An impedance bandwidth of $4.6 \mathrm{GHz}$ in 
the range $1.6-7.2 \mathrm{GHz}$ has been measured and acceptable radiation properties have been observed throughout it. The operating bandwidth of this novel antenna covers numerous communication systems. Moreover, thanks to its compact dimensions this radiating element allows appealing applications for indoor, vehicular and mobile purposes.

\section{ACKNOWLEDGEMENTS}

This work was supported by Science Foundation Ireland.

\section{REFERENCES}

[1] N. P. Agrawall, G. Kumar and K. P. Ray, Wide-band planar monopole antenna, IEEE Trans. Antennas Propag., vol. 46, no. 2, pp. 294-295, 1998.

[2] T. Tsukiji, Y. Kumon and M. Yamasaki, Double-folded monopole antenna using parallel line or coaxial cable, IEE Proc.-Microw. Antennas Propag., vol. 149, no. 1, pp. 17-22, 2002.

[3] Z.N. Chen, M.Y.W. Chia and M.J. Ammann, Optimization and Comparison of Broadband Monopoles, IEE Proc.- Microw. Antennas Propag., vol. 150, no. 6, pp. 429-435, 2003.

[4] M. J. Ammann and Z. N. Chen, A wideband shorted planar monopole with bevel, IEEE Trans. Antennas Propag., vol. 51, no. 4, pp. 901-903, 2003.

[5] S.-W. Su, K.-L Wong and C.-L. Tang, Ultra-wideband square planar monopole antenna for IEEE 802.16a operation in the 2-11 GHz Band, Microwave and Optical Technology Letters, vol. 42, no. 6, pp. 463-466, 2004.

[6] M. J. Ammann and Z. N. Chen, Investigation on L-shaped planar antennas, Radio Science, vol. 39, RS2009, 2004.

[7] C. Y. Chiu and C. H. Chan, A Wideband Antenna with a Folded Patch Feed, IEEE International Workshop on Antenna Technology, pp. 45-48, March 2005. 